Working Paper/Document de travail

2008-30

\title{
Non-Linearities, Model Uncertainty, and Macro Stress Testing
}

by Miroslav Misina and David Tessier 
Bank of Canada Working Paper 2008-30

September 2008

\title{
Non-Linearities, Model Uncertainty, and Macro Stress Testing
}

by

\author{
Miroslav Misina ${ }^{1}$ and David Tessier ${ }^{2}$ \\ ${ }^{1}$ Monetary and Financial Analysis Department \\ Bank of Canada \\ Ottawa, Ontario, Canada K1A 0G9 \\ mmisina@bankofcanada.ca \\ Université du Québec en Outaouais \\ david.tessier@uqo.ca
}

Bank of Canada working papers are theoretical or empirical works-in-progress on subjects in economics and finance. The views expressed in this paper are those of the authors.

No responsibility for them should be attributed to the Bank of Canada. 


\section{Acknowledgements}

The authors would like to thank Gino Cateau, Céline Gauthier, Pierre St-Amant, the participants at the Bank of Canada internal workshops, and the participants and discussants at the Bank of Canada's 2007 workshop 'Developing a Framework to Assess Financial Stability' for valuable comments and suggestions. 


\begin{abstract}
A distinguishing feature of macro stress testing exercises is the use of macroeconomic models in scenario design and implementation. It is widely agreed that scenarios should be based on "rare but plausible" events that have either resulted in vulnerabilities in the past or could do so in the future. This requirement, however, raises a number of difficult statistical and methodological problems. Economic models, as well as the statistical models of the relationships among economic variables, generally focus on capturing the average rather than the extreme behaviour, and frequently rely on the assumption of linearity. In this paper we show that these models are particularly ill-suited for stress-testing as they do not adequately capture past behaviour in extreme events, nor do they generate plausible responses to shocks under stress. Whereas one might argue that the use of these models is still preferable to no having no models, since they at least impose the consistency restrictions on the paths generated under the scenario, failing to deal with a large extent of uncertainty of these paths may lead to results that are non-informative, and potentially misleading. The paper illustrates both of these problems by a series of examples, but our conclusions have broader implications for the types of models that would be useful in these exercises.
\end{abstract}

JEL classification: C15, G21, G33

Bank classification: Financial stability

\title{
Résumé
}

La conduite de simulations de crise macrofinancière se distingue, entre autres, par l'emploi de modèles macroéconomiques dans la conception et le déroulement des scénarios. Il est généralement admis que ces scénarios doivent illustrer des événements « rares mais plausibles » qui ont déjà causé des vulnérabilités ou pourraient le faire. Cette condition pose toutefois certains problèmes statistiques et méthodologiques épineux. En effet, les modèles économiques, aussi bien que les modèles statistiques formalisant les relations entre les variables économiques, tendent à reproduire des évolutions ordinaires plutôt qu'extrêmes, et sont souvent construits à partir d'une hypothèse de linéarité. Les auteurs montrent que ces modèles sont très mal adaptés à la conduite de simulations de crise, parce qu'ils sont incapables de bien restituer les évolutions passées lors d'événements extrêmes et de produire des réactions crédibles aux chocs générés par les crises. Si d'aucuns estiment que, dans la mesure où ils soumettent au moins les trajectoires scénarisées à un critère de cohérence, de tels modèles valent mieux que rien, il reste que le fait de ne pas tenir compte de l'importante marge d'incertitude qui entoure les prévisions risque de mener à des résultats sans valeur informative, voire trompeurs. Les auteurs illustrent chacun de ces problèmes 
par une série d'exemples. De façon plus générale, leurs conclusions ont cependant des implications quant aux modèles susceptibles de servir aux simulations étudiées.

Classification JEL : C15, G21, G33

Classification de la Banque : Stabilité financière 


\section{Introduction}

Stress testing, at its most general level, is an investigation of the performance of an entity under abnormal operating conditions. A key part in any stress-testing exercise is a decision on what constitutes abnormal operating conditions, which is part of the scenario design. Whereas the implementation of a scenario by using an economic model is, arguably, a well-defined technical exercise, selection of scenarios is to a large extent an art that involves both an understanding of the past sources of stress, and forming an idea of what may cause problems in the future. Indeed, the foremost criterion in scenario design is that it be based on rare but plausible events that have either resulted in vulnerabilities in the past or could do so in the future. This criterion leads to a number of challenges, both statistical and methodological, the most prominent of which are the examined in this paper.

Finding suitable statistical models that capture rare events of the past, assuming that such events are in the sample, is a difficult exercise. Standard statistical models, such as regression models, while well-suited to characterize the average behaviour treat extreme realizations as outliers. On the other hand, the use of specialized techniques (for example, extreme value theory) is dependent on having a minimum number of realizations of extreme events in the sample. Small number of such realizations may result in instability of parameter estimates.

Implementation of scenarios based on extreme events using standard economic models imposes a burden on these models they were never meant to bear. Standard macroeconomic models are typically designed to provide an explanation of 'normal times', or medium-term cyclical fluctuations. These models are typically 
linearized around a steady state, which severely limits the types of responses to shocks that can be obtained, and makes them ill-suited for implementation of scenarios based on extreme events. In addition, the presence of built-in stabilizing mechanisms (optimal policy responses, for example) tends to dampen the impact of shocks in these models and necessitates the use of extreme shocks to produce the impact that significantly deviates from the equilibrium behaviour they are constructed to describe.

One might argue that even though not well-suited for the problem at hand, it is still preferable to use models because (i) they are the best tools we have, and (ii) they at least ensure that the scenarios are internally consistent. Internal consistency is certainly desirable, but the models built to explain normal times may yield a very poor, even if consistent, approximation to what might happen under extreme circumstances. Practically, this means that the confidence intervals around our scenario paths will be quite large, reflecting a great degree of uncertainty regarding the location of the internally consistent responses under stress. Whereas it is clear that ignoring this uncertainty will lead to generally misleading assessments of the impact of the scenario, it is far less clear how this uncertainty should be taken into account.

How prevalent are these problems in the literature? Since macro stress testing is a relatively new area, there is no consensus on the set of tools, or the best approach to use. There are a variety of approaches, and some examples in the area of credit risk macro stress-testing include Jimenez and Mencia (2007), Misina, Tessier, and Dey (2006), Segoviano and Padila (2006), Sorge and Virolainen (2005), Virolainen (2004), and Wilson (1997a,b). These papers have two key features in common: (i) use of forecasted macroeconomic variables to charac- 
terize the impact of shocks on the economy, and (ii) the need to translate these macro variables into loss distributions, usually done by mapping them into defaults under stress. The most straightforward approach is to use the forecasted paths of the macroeconomic variables without taking into account forecast risk, and map these variables into defaults by assuming a linear relationship. Whereas there is some variation among the papers in terms of the treatment of macro uncertainty (Misina et al (2006) do not take it into account; Segoviano and Padilla (2006) seems to implicitly take it into account in estimating the joint distribution of default rates), the linear specification (possibly with some lags) is the norm.

The objective of our paper are two-fold:

1. to illustrate the impact of linearity on the features of the responses to shocks, and how a simple non-linear specification can result in improvements,

2. to illustrate the consequences of ignoring the uncertainty associated with the forecasts coming out of the macro models.

The illustrations are done in the context of macro stress-testing model used in Misina et al (2006), which is based on Virolainen (2004). The issues, however, are more general, and our results lead to a characterization of the key features of the models that would be useful for performing the stress-testing exercises. In our examples, non-linearities and model uncertainty are taken into account in a particular way. These methods have their own limitations, and we do not view them as preferred solutions, but rather as the initial steps that should motivate a more in-depth investigation of these problems and a comparative analysis of various ways to deal with them. 
To facilitate the exposition, Section 2 contains a brief overview of the framework on which the macro stress-testing exercises are typically based. Section 3 deals with the assumption of linearity and its implications. In section 4 we focus on the issue of model uncertainty and its impact on the stress testing results. In the concluding section we bring together the results and trace their broader implications.

\section{A brief overview of the stress-testing framework}

A stylized representation of the framework commonly used in macro stress testing exercises is shown in Figure 1.

\section{FIGURE 1}

The framework consists of three parts:

- Macroeconomic module (Block 1)

- 'Transmission mechanism' between macro variables and financial institutions' portfolios (Block 2)

- Loss assessment module (Block 3)

The implementation of the scenario is done in Block 1. The events selected are typically translated into statements about the nature and magnitude of shocks which are then applied to a macroeconomic model The output of Block 1 is a series of paths of macroeconomic variables under stress. In Block 2 , the impact of the shocks, in the form of the path of the macroeconomic variables under stress, 
is related to financial institutions' portfolio. ${ }^{1}$ The output of Block 2 is used as input in Block 3 in which losses are assessed by computing loss distributions and various statistics of interest. ${ }^{2}$

\section{Linearity and stress testing}

The consequences of linearity can be illustrated in different ways. One could either work directly in Block 1, or focus on the transmission mechanism in Block 2 , treating it as a by-product of the specification in Block 1. We opt for the latter, since this allows us to illustrate the main issues in a particularly simple setting.

Given that our examples are based on credit risk stress testing, the transmission mechanism in Block 2 is quite specific: it is a model of the relationship between the macroeconomic variables and sectoral default rates. In this setting, we show how the functional form used in specification of default regressions affects the nature of the responses of default probabilities under stress. In particular, we argue that the assumption of linear relationship imposes severe restrictions on the responses of default probabilities to macroeconomic shocks. These restrictions are particularly undesirable in stress-testing exercises. To remedy this problem, we introduce non-linearities in a simple but effective way, and illustrate their impact on responses by a series of examples.

\footnotetext{
${ }^{1} \mathrm{~A}$ common way of implementing this in credit risk stress testing is to relate macroeconomic variables to default probabilities. Chan-Lau (2006) surveys different approaches to estimating default probabilities.

${ }^{2}$ The separation between Blocks 1 and 2 is not based on a fundamental independence between macro modelling and the modelling of default probabilities, but is rather a reflection of a lack of integrated models that could be used in this setting.
} 
In the following discussion the following notation is used:

- $x$ - a set of macroeconomic variables. The relationships among these variables are modelled in Block 1

- $\pi$ - default probability. When working with sectors, it can be interpreted as the average default probability in a sector.

- $L$ - loss distribution.

\subsection{General discussion}

Relating default probabilities and macroeconomic variables in Block 2 amounts to selecting the functional form in the expression

$$
\pi=f(x) .
$$

Specifying $f$ as a linear function is a simple solution that has a number of undesirable consequences. To see this, consider the following example in which $\pi=a x$. The impact of changes in $\mathrm{x}$ is given by

$$
\frac{d \pi}{d x}=a .
$$

This simple expression makes it clear that the restrictions on responses imposed by linear models are rather severe, with the following properties:

- symmetry: the magnitude of responses is the same regardless of whether the shock is positive or negative. 
- proportionality: responses are proportional to the change in the exogenous variable.

- history independence: the shape of responses is independent of initial conditions of the macroeconomic variables

None of these restrictions are appealing in the context of stress testing exercises, where asymmetry, non-proportionality, and history dependence would seem to be desirable properties. For example, one would expect a negative shock to have a different impact on companies depending on whether the economy is in a recession or in an expansionary phase.

Stress tests generally select scenarios that are severe but plausible, which implies that experimental shocks are usually quite large. With shocks of such magnitude, linear approximations to a possibly non-linear process might prove to be particularly poor.

To get response profiles with features more suitable for stress testing, the assumption of linearity has to be relaxed. A simple way to do it is to introduce higher-order terms, while preserving additivity. The following non-linear specification,

$$
\pi=a x+b x^{2}+c x^{3},
$$

delivers the response function

$$
\frac{d \pi}{d x}=a+2 b x+3 c x^{2},
$$

which generates asymmetric, non-proportional, and history-dependent responses. This type of response function would imply that the impact of shocks would differ in good and bad states, both qualitatively and quantitatively. Jorda (2005) shows 
that this functional form approximates a wide range of classes of non-linear models reasonably well.

\subsection{Illustrative examples}

The examples in this section build on the linear specification of default probability regressions in Misina et al (2006). In that paper, sectoral default probability regressions take the form

$$
\ln \left(\frac{\pi_{t}}{1-\pi_{t}}\right)=\mu+\sum_{l=1}^{L} \beta_{l} X_{t-l}+e_{t}
$$

where $l$ denotes the number of lags.

The explanatory variables are Canadian macroeconomic variables (real GDP, real interest rate, unemployment rate) and their lags. One way to introduce nonlinearities is to retain additivity but include higher-order terms:

$$
\ln \left(\frac{\pi_{t}}{1-\pi_{t}}\right)=\mu+\sum_{l=1}^{L} \beta_{l}^{(1)} X_{t-l}+\sum_{l=1}^{L} \beta_{l}^{(2)} X_{t-l}^{2}+\sum_{l=1}^{L} \beta_{l}^{(3)} X_{t-l}^{3}+e_{t} .
$$

The key advantages of introducing non-linearities in this manner are simplicity and flexibility. Addition of other variables and higher-order terms does not represent econometric difficulties, as the relationship remains linear in parameters.

The data used to estimate these regressions are Canadian real GDP growth rate (year-over-year), real interest rate on medium-term business loans, the unemployment rate, and sectoral default rates. The data spans the period from 1987:1 to $2005: 4 .^{3}$

\footnotetext{
${ }^{3}$ Long time series on default rates are generally not available. Misina and Tessier (2007) describe how such series were constructed for Canada.
} 
To examine the impact of the introduction of non-linearities, we focus on the behaviour of predicted sectoral default rates following the early 1990s recession in Canada, which occurred between 1990:4 and 1991:3. The forecasts are given for the 3-year period starting in 1991:4. ${ }^{4}$ Figure 2 contains the paths of historical and predicted default rates, where the latter are estimated by linear and non-linear models. ${ }^{5}$ The benefit of non-linearities is particularly evident in this stressful period, when the default rate reached its historical peak. The non-linear model captures this period much better than the linear one.

\section{FIGURE 2}

To get a better sense of the limitations of the linear model we perform two sets of experiments: (i) a change in the severity of recession, and (ii) a change in the initial conditions. The experiments are performed by exogenously changing Canadian GDP over the 1990:4 - 1991:3 period, and deriving the implications for the GDP, interest rate, and unemployment rate in the subsequent period using a three-variable vector autoregression model. ${ }^{6}$

\subsubsection{Change in the severity of recession}

In this experiment, we assume that the recession is very mild (10\% of the 1990/91 recession). This is implemented by multiplying the observations in the 1990:4 - 1991:3 period by 0.1. Everything else being the same, this should result in a

\footnotetext{
${ }^{4}$ Our specification includes four lags which fully take into account the period 1990:4 to 1991:3.

${ }^{5}$ Here, we show the results for the manufacturing sector only. The results for other sectors are qualitatively similar.

${ }^{6}$ The method used to accomplish this is explained in the Appendix.
} 
significant decrease in default rates predicted by the model.

\section{FIGURES 3,4}

Figures 3 and 4 contain the results for linear and non-linear models, respectively. In both figures, we compare the default rate paths predicted under the 1990/91 recession to the paths predicted under our much milder hypothetical recession. The non-linear model is clearly more responsive than the linear one, and the difference is more important the larger the shock. The key reason is that, in contrast to the linear model, the non-linear model is not bound by the assumption of proportionality.

\subsubsection{Change in the initial conditions}

In this experiment we change the conditions prior to the recession by converting them from unfavourable (approximately zero percent GDP growth rate) to favourable (3.7 percent GDP growth rate). Starting from these new conditions, we reduce the GDP growth rate by the magnitude observed in the 1991 recession, so that the decline in GDP growth stays the same at -3.2 percent. Notice, however, that the lowest GDP growth rate in this experiment is 0.5 percent, rather than -3.4 percent observed in 1991. One would expect that, starting from these new initial conditions, the same magnitude of changes in GDP growth would have a much smaller impact, since favourable economic conditions put companies in a better position to absorb these shocks.

\section{FIGURES 5,6}


Figures 5 and 6 contain the results for linear and non-linear models. In both cases, there is a decline in default rates relative to the original setting, but it is much more significant in the case of non-linear model. Indeed, this model now predicts only a slight change in default rates, while the responses in the linear model are limited to an approximately parallel shift down. ${ }^{7}$ This example highlights the invariance of the shape of the response in the linear specification to changes in initial conditions.

One implication of this result is that if the initial conditions are favourable, a much bigger decline in GDP would be needed to induce the response of default rates comparable to that observed in 1991 recession.

\section{Model uncertainty and stress testing}

Model uncertainty is an issue that is familiar to risk managers, and there are various ways in which individual financial institutions deal with it. ${ }^{8}$ In macro stress testing, macroeconomic model uncertainty is a particular aspect of the uncertainty associated with these types of exercises. The issue does not seem to have received much attention in the literature, and it is difficult to deal with at a general level. We will examine it by mapping it into the risk associated with the

\footnotetext{
${ }^{7}$ The shift would be exactly parallel if the changes in all explanatory variables were fixed exogenously, which is not the case in our model.

${ }^{8}$ See, for example, Crouhy et al. (2006), Chapter 14, for a discussion. Even though the issues described there are referred to as model risk, strictly speaking the discussion is about model uncertainty. The difference between the two is in the Knightian distinction of the outcomes to which probabilities can be assigned (risk), and the ones in which this is not possible (uncertainty).
} 
forecasts coming out of the macroeconomic models specified in Block $1 .^{9}$ In this context, we will illustrate that the problem cannot be ignored and that, in certain circumstances, forecast risk may drive the results of stress tests.

\subsection{General discussion}

Let $L$ denote the losses that can arise out of the scenario. The objective of the loss assessment block is to obtain the distribution of losses as a function of the outcomes arising under the scenario. The magnitude of losses will depend on default rates, exposures, and losses given default. Assuming for simplicity that the last two are fixed, the distribution of $L$, denoted $\phi(L)$, will depend only on default rate, $\pi$, which is a function of the macro variables.

To illustrate the nature of the problem, suppose that

$$
\pi=\mathbf{X} \boldsymbol{\beta}+\varepsilon,
$$

where $\mathbf{X}$ is a matrix of macro variables. To generate the loss distribution we need a set of possible forecasted values of $\pi$ conditional on a given path of macro variables, $\mathbf{X}_{0}$, generated by the macro model. If $\mathbf{X}_{0}$ is assumed to be deterministic, the forecast variance will be given by

$$
\sigma_{\varepsilon}^{2}+\mathbf{X}_{0}^{\prime}\left(\sigma_{\varepsilon}^{2}\left(\mathbf{X}^{\prime} \mathbf{X}\right)^{-1}\right) \mathbf{X}_{0}
$$

Macroeconomic model uncertainty can be viewed as the uncertainty regarding the path of the macro variables under the scenario. The implication of our earlier observations regarding the performance of typical macro models under extreme

\footnotetext{
${ }^{9}$ Here, by forecasts we mean the paths of the endogenous variables in the macro model conditional on the realization of pre-specified exogenous shocks.
} 
shocks, and the possibility of changes in structural relationships during times of stress, is that there is a great deal of uncertainty associated with the paths of macro variables. Consequently, the assumption of deterministic $\mathbf{X}_{0}$ is tantamount to ignoring this type of uncertainty.

To take macro uncertainty into account, it is necessary to replace the assumption of deterministic $\mathbf{X}_{0}$ by the assumption that it is stochastic. Although the precise impact is difficult to ascertain, there seems to be agreement that when $\mathbf{X}_{0}$ is stochastic (2) will underestimate the true variance. ${ }^{10}$ Greater variance of forecasts will imply a greater variance in losses, with more mass in the tails of the loss distribution that would be obtained under the assumption that the path of macro variables in certain. In addition, given that the forecast risk increases with the horizon, increases in the stress testing horizon would lead to an increased divergence between the results that take macro forecast risk into account and the ones that do not.

Whether it is appropriate to ignore macro uncertainty or not, depends on the type of question a particular stress test is designed to answer. If the objective is to answer the question "What are the losses if variable $x$ equals a specific value $x_{0}$ ?", the issue of macro uncertainty is not present. These types of questions are typically asked in 'single-factor' stress tests.

If the question is "What are the losses in a set of macro shocks were to materialize?", and if answering that question involves the use of a macro model to generate the paths of macro variables under stress, the question of model uncertainty cannot be avoided. ${ }^{11}$

\footnotetext{
${ }^{10}$ See Greene (2003), Section 6.6 for a discussion and references.

${ }^{11}$ There is a large literature in macroeconomics that deals with the issue of model uncertainty,
} 


\subsection{An example}

To illustrate the above ideas, we perform a simple exercise, and compute expected losses and the $99.9 \%$ value-at-risk with and without macroeconomic forecast risk. In this exercise, we continue to use the 1990-1 recession as a benchmark. Taking the initial conditions as the actual conditions that prevailed in the 1989:4 to 1990:3 period, we forecast the key macroeconomic variables using our macro model, and then compute default rates consistent with these forecasts. To analyze the impact of macro risk on the losses, we generate loss distributions with and without macro risk. The measure of forecast risk is standard errors of forecasts coming from the macro model.

Loss distributions are generated in the following way:

1. Generate a path for the macroeconomic variables using a macroeconomic model, ${ }^{12}$

2. Generate a vector of $s$ random variables using the variance-covariance matrices $\hat{\boldsymbol{\Sigma}}_{\mathbf{e}_{\mathbf{X}}}$ and $\hat{\boldsymbol{\Sigma}}_{\mathbf{e}_{\pi}}$. This is done by first generating a vector $\mathbf{Z} \sim N(\mathbf{0}, \mathbf{I})$, and then calculating $\hat{\mathbf{e}}_{t}=A^{\prime} Z_{t}, \hat{\mathbf{e}}_{t} \sim N(\mathbf{0}, \hat{\Sigma})$, where $\hat{\boldsymbol{\Sigma}}=A A^{\prime}$, and $A$ is obtained via Cholesky decomposition of the variance-covariance matrix.

3. Substitute the results of the previous two steps into equation (1) to obtain the values of default probabilities for each industry at a given point in time.

4. For each value of simulated default probability for industry $s$, compute the

and decision-making in such environments. Hansen and Sargent (2007) provide an overview.

${ }^{12}$ The details of the model are provided in the appendix. 
expected loss in that industry according to

$$
\widetilde{E L}_{t}^{s}=\tilde{\pi}_{t}^{s} \times e x_{t}^{s} \times l_{t}^{s} .
$$

The expected loss at time $t$ on the portfolio is computed by summing the expected losses in all industries:

$$
\widetilde{E L_{t}}=\sum_{s=1}^{S} \tilde{\pi}_{t}^{s} \times e x_{t}^{s} \times l_{t}^{s} .
$$

To obtain a loss distribution, a large number of realizations of $\tilde{\boldsymbol{\pi}}_{t}$ are generated and, for each realization, steps (i)-(iv) are repeated.

\subsubsection{Results}

Figures 7 presents the loss distribution under non-linear specification when there is no risk about macroeconomic forecasts. Figure 8 contains the same information when the risk about macroeconomic forecasts is taken into account. The distributions are computed at 2-years horizon.

\section{FIGURE 7,8}

Taking into account macroeconomic forecast risk yields a loss distribution that is skewed and has heavier tail than the standard case. This implies that valueat-risk results under these two distributions can be quite different. Indeed, in the cases shown in these two figures the 99.9 percent value-at-risk is 4.2 and 8.3 percent, respectively. Such difference in magnitudes will have large impact on the computation of regulatory capital and the capital adequacy ratio. 
Figures 7 and 8 present results at a point in time. To get a sense of the behaviour of losses over time, we have computed expected and $99.9 \%$ value-atrisk (unexpected losses) at each point over the 4-years stress-testing horizon. The results are shown in Figure 9.

\section{FIGURE 9}

We first observe that the introduction of macroeconomic forecast risk does not impact the expected losses. This is not surprising, since expected losses are computed using expected default rates, which, in turn, depend on the forecasted values of the macro variables.

The difference between unexpected losses in two cases is due exclusively to forecast risk which is increasing over time. The shape of the unexpected losses curve, however, will be determined by both the behaviour of the default rates, and the extent of forecast risk. The latter is increasing with the horizon, and, everything else being the same, one would assume that unexpected losses would be monotonically increasing. As Figure 9 shows, this is clearly not the case. The reason is that the second determinant of losses-the level of default rates-is not

the same. In contrast to forecast risk which monotonically increases with the forecast horizon, default rates will typically be increasing to begin with, and after reaching a peak, will tend to decrease. Taking these factors into account yields the following general characterization of the behaviour of unexpected losses:

- at shorter horizons, the behaviour of default rates and forecast risk will reinforce each other, leading to a continuous increase in unexpected losses.

- at longer horizons, after default rates have peaked, the impact on the un- 
expected losses will depend on the importance of increasing forecast risk, relative to the trend in default rates.

We observe both of these stages in Figure 9. In the first 8 periods the two determinants reinforce each other, while after the peaks the outcome depends on their interaction.

Of course, the nature of the results will be strongly affected by the functional form used to model default rates. In the above examples, we used the nonlinear specification of Section 2. Figure 10 contains a comparison of these results to the ones obtained with a linear specification using the same variables. Not surprisingly, non-linearities magnify the impact of forecast risk.

\section{FIGURE 10}

\section{Summary and conclusions}

In this paper we examined the importance of non-linearities and macroeconomic uncertainty in macro stress testing exercises. These exercises have two key features: (i) use of forecasted macroeconomic variables to characterize the impact of shocks on the economy, and (ii) the need to translate these macro variables into loss distributions, usually done by mapping them into defaults under stress. The most straightforward approach is to use the forecasted paths of the macroeconomic variables without taking into account forecast risk, and map these variables into defaults by assuming a linear relationship. Neither choice is satisfactory: the assumption of linearity will deliver the response profiles which are not well suited to the main objective, and ignoring the forecast risk will lead to underestimation 
of the value-at-risk.

The non-linear specification used in this paper is a simple one, but even in this simple case the benefits are clear. Of course, the importance of non-linearities will depend on the nature of the sample and the incidence of stressful episodes. Even when there is only one stressful episode in the sample, the non-linear terms may capture it well, but the robustness of the specification will inevitably be an issue. To fully assess the extent of the problem, if any, a sample with more than one stressful episode would be needed, but the nature of the sample is an exogenously given constraint rather than a matter of choice.

Taking into account model uncertainty is more difficult, and its reduction to forecast risk is, ultimately, unsatisfactory. Nonetheless, even at that level of analysis, the impact on the results is clear, although the feasibility of doing that will depend on the types of macroeconomic models used. In this paper we used a simple statistical model, which makes the generating the forecasts and assessing forecast errors straightforward. The drawback of these models is that they lack behavioural foundations, and can, at best, be thought of as reduced forms of some unspecified behavioural models, but this may not be entirely satisfactory. One solution is to use behavioural models, but that may lead to a different set of problems. ${ }^{13}$ It is clear, however, that no matter how sophisticated the macroeconomic model, forecast risk is non-decreasing with the forecast horizon, and may, under some circumstances, become the key factor determining the magnitude of losses. This is not necessarily desirable, and the issue of the length of the horizon should be carefully considered in the design of these exercises.

\footnotetext{
${ }^{13}$ In behavioural macro models that are calibrated rather than estimated, producing forecasts and assessing forecast errors is a challenge, although this is an area of active research.
} 
More generally, the analysis of this paper points to the features that the macroeconomic models that would be useful in stress testing should possess. They should explicitly characterize the mechanism giving rise to non-linearities, and also produce an estimate of the errors associated with the forecasts coming out of these models. Recent work by Goodhart et al (2006) and Alessandrini et al (2007) are the examples of different types of work that are, broadly speaking, in line with the suggestions of this paper. 


\section{References}

[1] Alessandrini, P., P. Gai, S. Kapadia, N. Mora, and C. Puhr. 2007. "A framework for quantifying systemic stability." Bank of England mimeo.

[2] Blasche, W., M. Jones, G. Majnoni, and S. Peria. 2001. "Stress testing of financial systems: An overview of issues, methodologies, and FSAP experiences." IMF Working Paper 01-88.

[3] Chan-Lau, J. 2006. "Fundamentals-based estimation of default probabilities: A survey." IMF Working Paper 06-149.

[4] Crouhy, M, D. Galai, and R. Mark. 2006. The Essentials of Risk Management. McGraw-Hill.

[5] Goodhart, C., P. Sunirand, and D. Tsomocos. 2006. "A model to analyze financial fragility." Economic Theory 27: 107-142.

[6] Greene, W. 2003. Econometric Analysis, Fifth Edition. Prentice Hall.

[7] Hansen, L. P., and T. Sargent. 2007 Robustness. Princeton University Press.

[8] Jimenez, G. and J. Mencia. 2007. "Modelling the distribution of credit losses with observable and latent factors." Bank of Spain Working Paper No. 0709.

[9] Jorda, O. 2005. "Estimation and inference of impulse responses by local projections." American Economic Review 95, 1: 161-182.

[10] Misina M., D. Tessier, and S. Dey. 2006. "Stress-testing the corporate loans portfolio of the Canadian Banking sector." Bank of Canada Working Paper 2006-47. 
[11] Misina, M. and D. Tessier. 2007. "Sectoral default rates under stress: The importance of non-linearities." Bank of Canada Financial System Review (June): 49-54.

[12] Segoviano, M. and P. Padilla. 2006. "Portfolio credit risks and macroeconomic shocks: Applications to stress-testing under data-restricted environments." IMF Working Paper 06-283.

[13] Sorge, M, and K. Virolainen. 2006. "A comparative analysis of macro stresstesting methodologies with application to Finland." Journal of Financial Stability 2 (2): 113-151.

[14] Virolainen, K. 2004. "Macro stress testing with a macroeconomic credit risk model for Finland." Bank of Finland Discussion Paper No. 18.

[15] Wilson, T. 1997a. "Portfolio credit risk (I)." Risk 10(9): 11-119.

[16] Wilson, T. 1997b. "Portfolio credit risk (II)." Risk 10(10): 56-61. 


\section{Appendix: A brief description of the macroeconomic model}

In this appendix, we briefly describe the dynamic model that has been used in Section 3.

In our framework, we suppose that the set of macro variables follow a linear autoregressive process. For each of these macro variables, the objective is to obtain a path of predicted value conditional to a set of initial conditions. ${ }^{14}$ These can be calculated in different ways. A natural strategy consists in estimating a model to characterize the dependence structure of successive observations, from which the future observations can be computed recursively to obtain the optimized multi-step predictions.

One of the most common examples of that strategy consists in specifying a VAR model under which the multi-step forecast are obtained through the dynamic simulations. Another strategy is to specify direct forecasting models that are reestimated for each horizon. The idea behind is to replace these dynamic simulations by a set of sequential regressions of the endogenous variable shifted several steps ahead. Hence, we can specify a model that allows for projection of the vector $\mathbf{X}_{t+h}$ onto the linear space spanned by the information available at time $t$ :

$$
\mathbf{X}_{t+h}=\mu+\sum_{k=1}^{p} \pi_{k}^{(h)} \mathbf{X}_{t+1-k}+\mathbf{e}_{t+h} .
$$

It is important to note that the matrices of coefficients $\pi_{k}^{(h)}$ depend on the horizon $h$ and will have to be reestimated at each horizon. The paths of the variables in our model (historical and forecasted) are shown in Figures B.1 to B.3.

\footnotetext{
${ }^{14}$ In our case, the initial conditions are those prevailing one year before 1991 recession (89Q490Q3).
} 


\section{Figures}

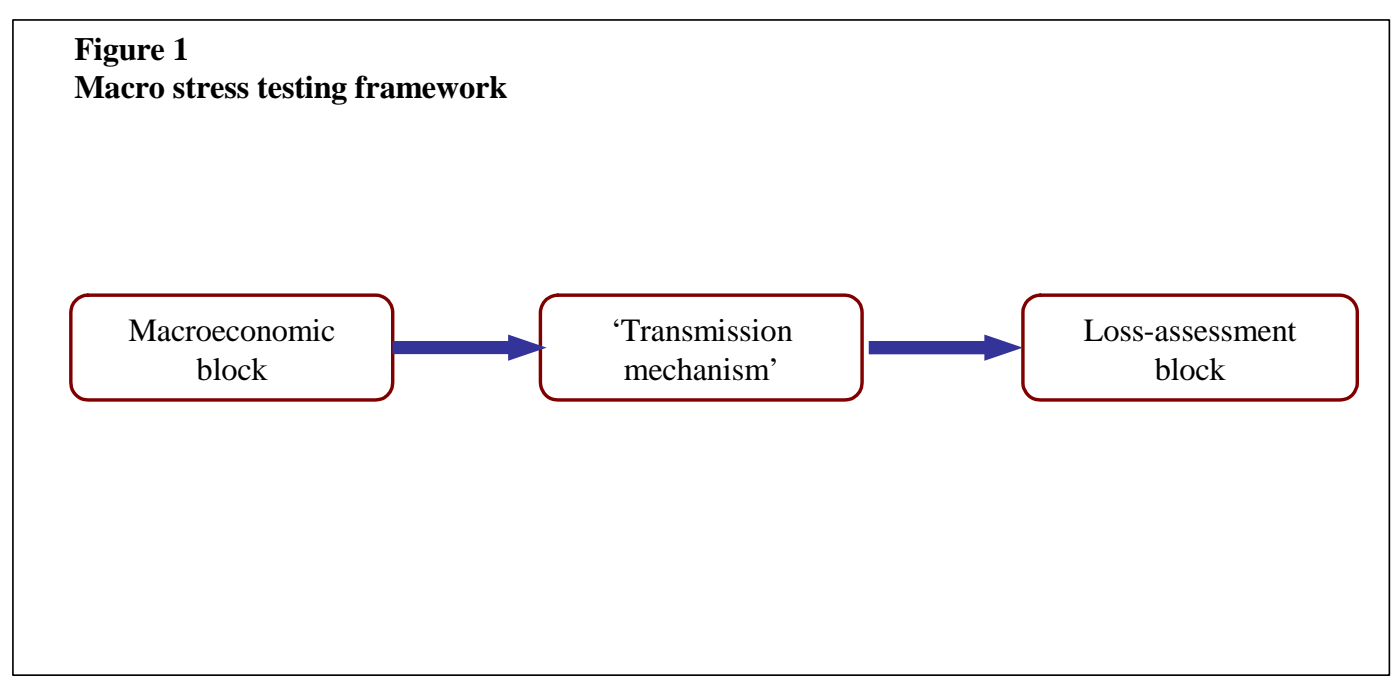

Figure 2

Historical and predicted default rates: Manufacturing sector

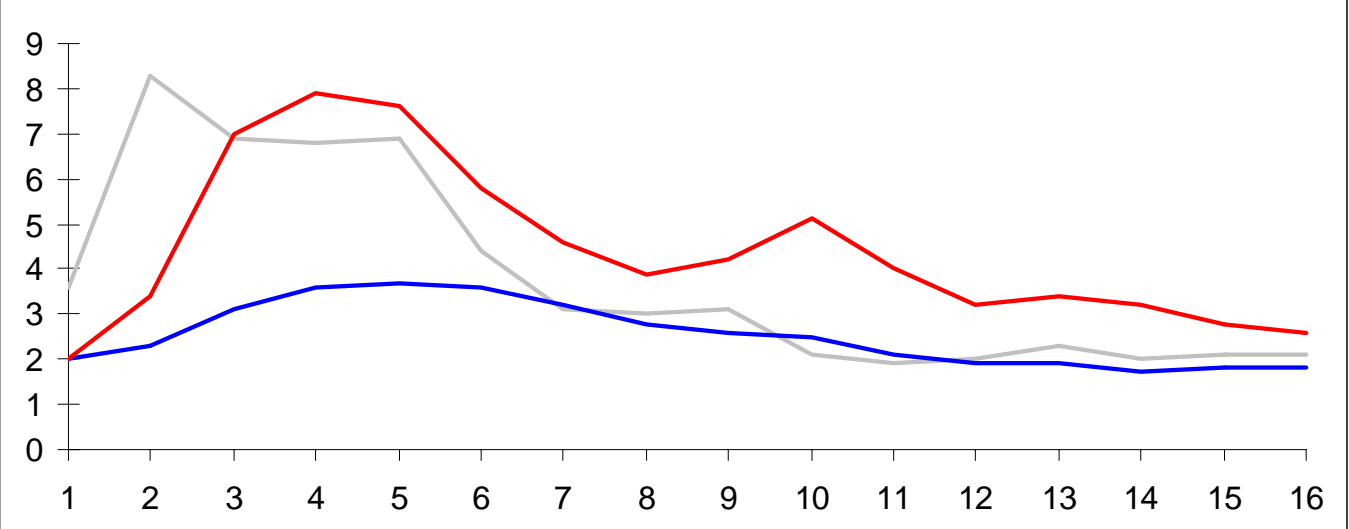

- Historical default rate

— Predicted default rate (linear model)

Predicted default rate (non-linear model) 
Figure 3

Impact of a change in the severity of recession on default rate:

Linear model

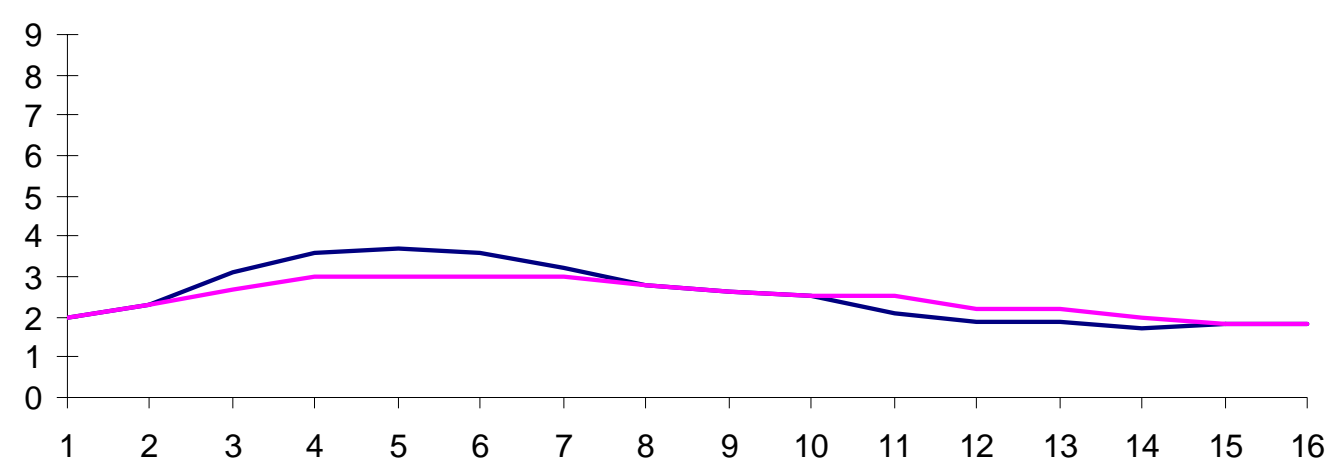

_ Predicted default rate (1990-1991 recession)

_ Predicted default rate (milder recession)

Figure 4

Impact of a change in the severity of recession on default rate:

Non-linear model

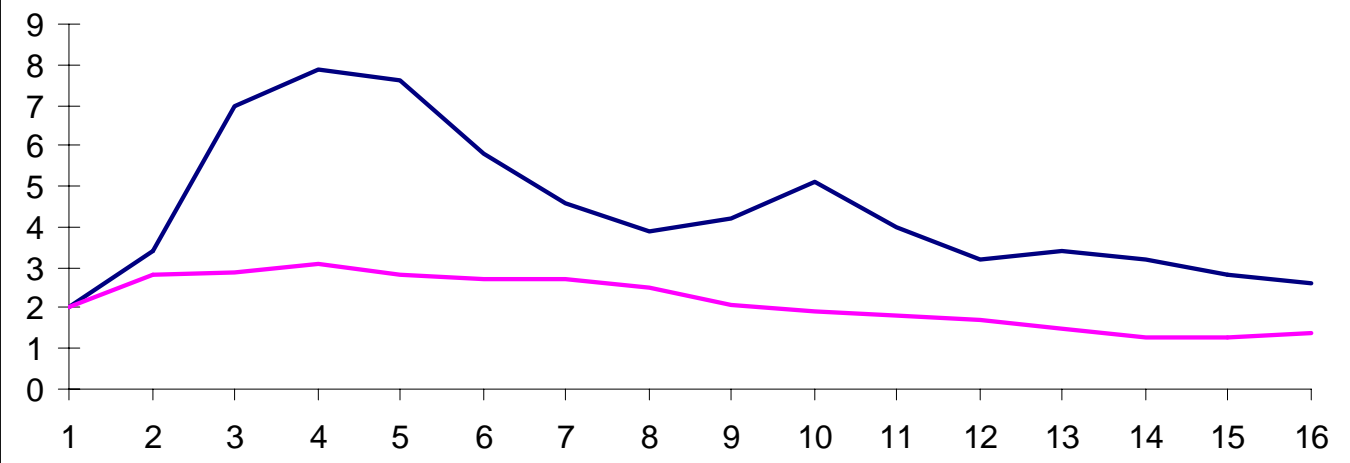

— Predicted default rate (1990-1991 recession)

_ Predicted default rate (milder recession) 


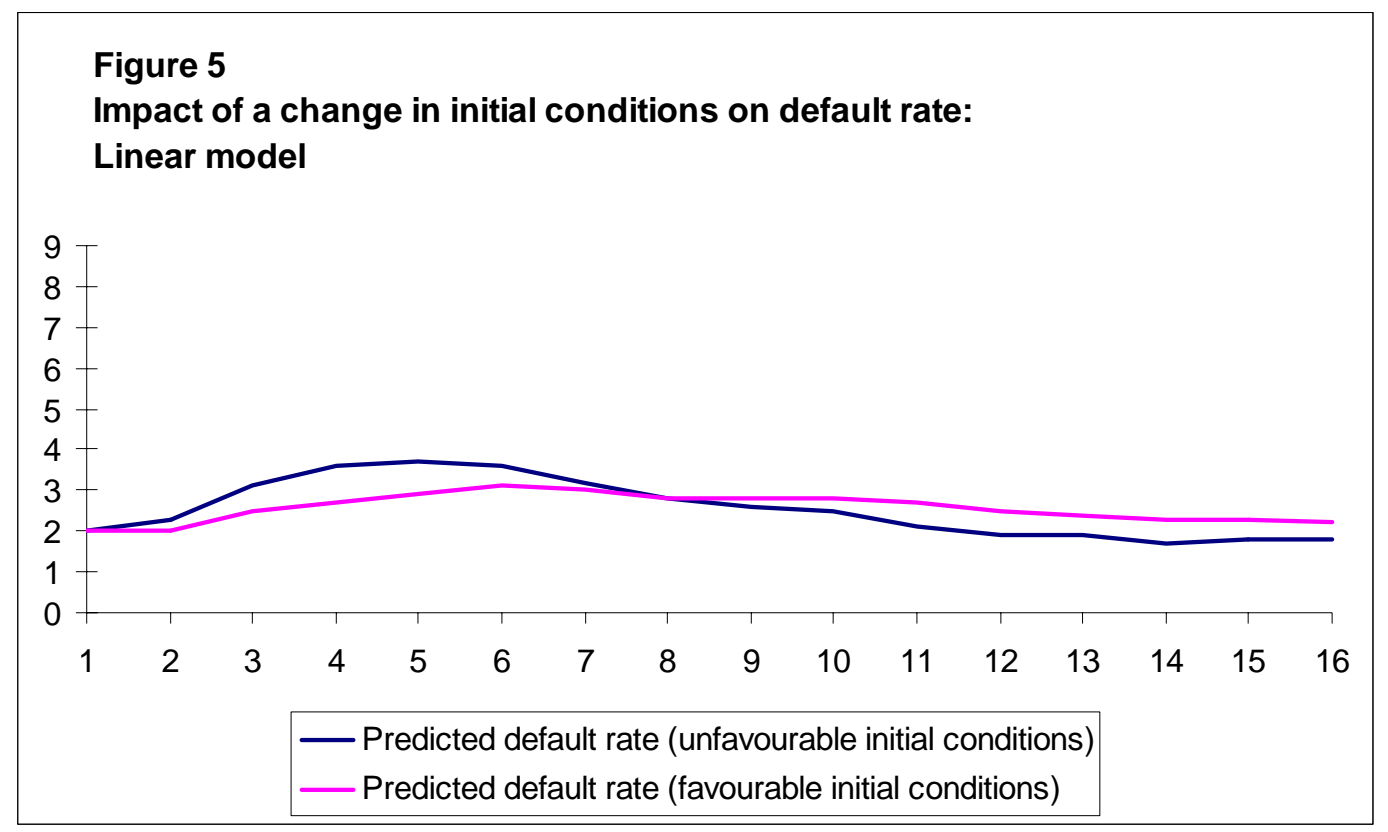

\section{Figure 6}

Impact of a change in initial conditions on default rate:

Non-linear model

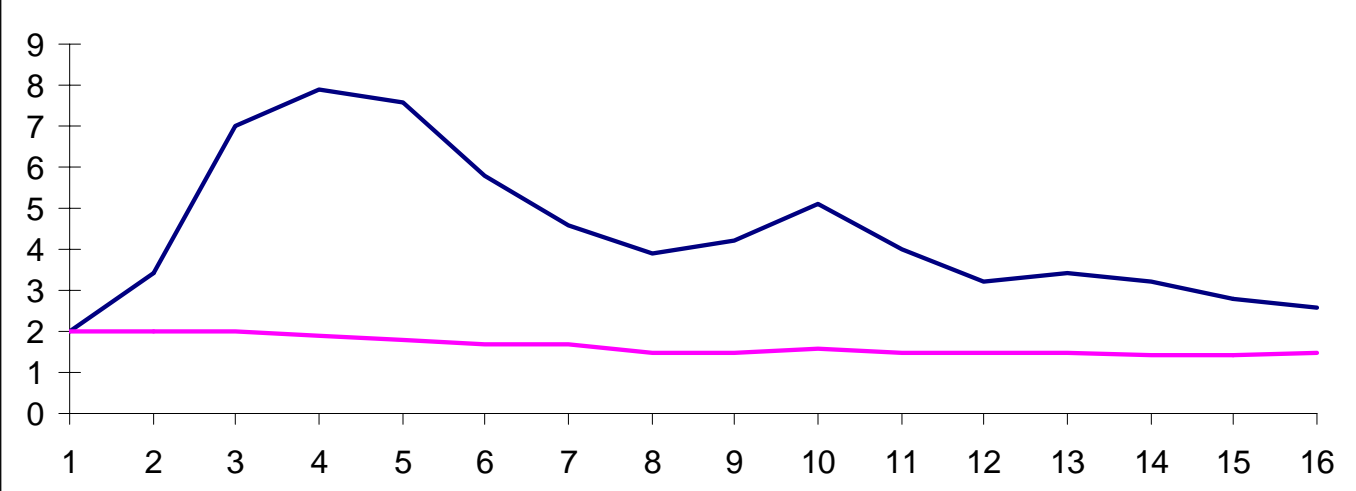

- Predicted default rate (unfavourable initial conditions) Predicted default rate (favourable initial conditions) 
Figure 7

Loss distribution without forecast uncertainty

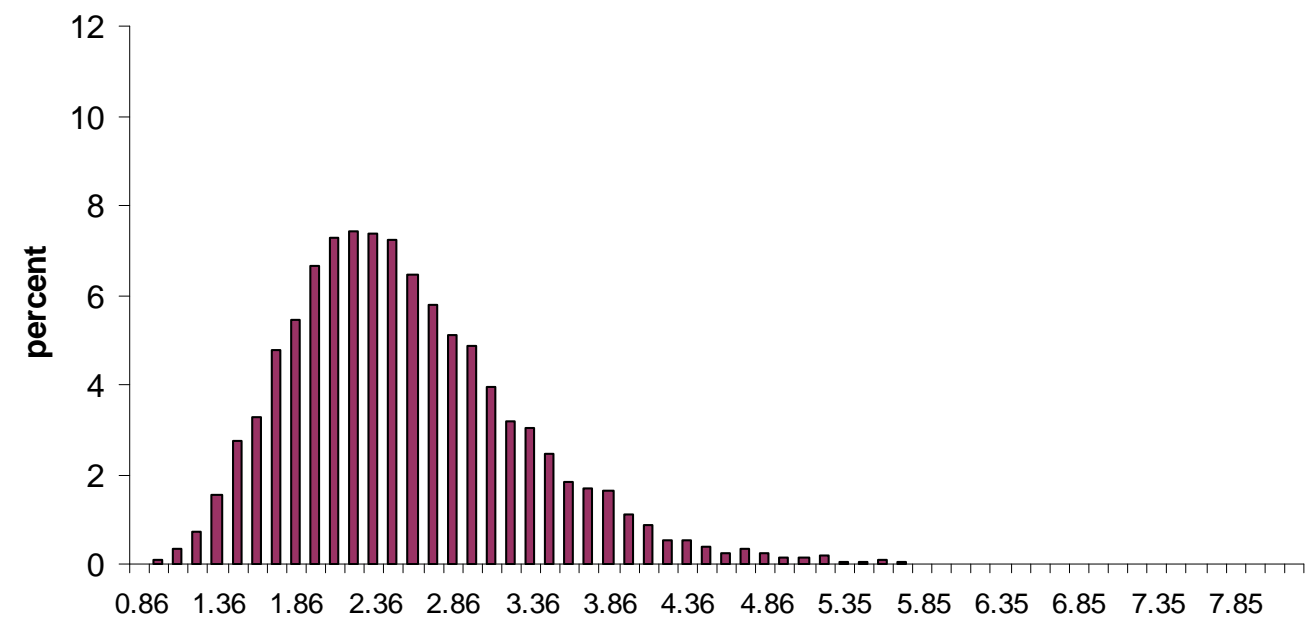

Figure 8

Loss distribution with forecast uncertainty

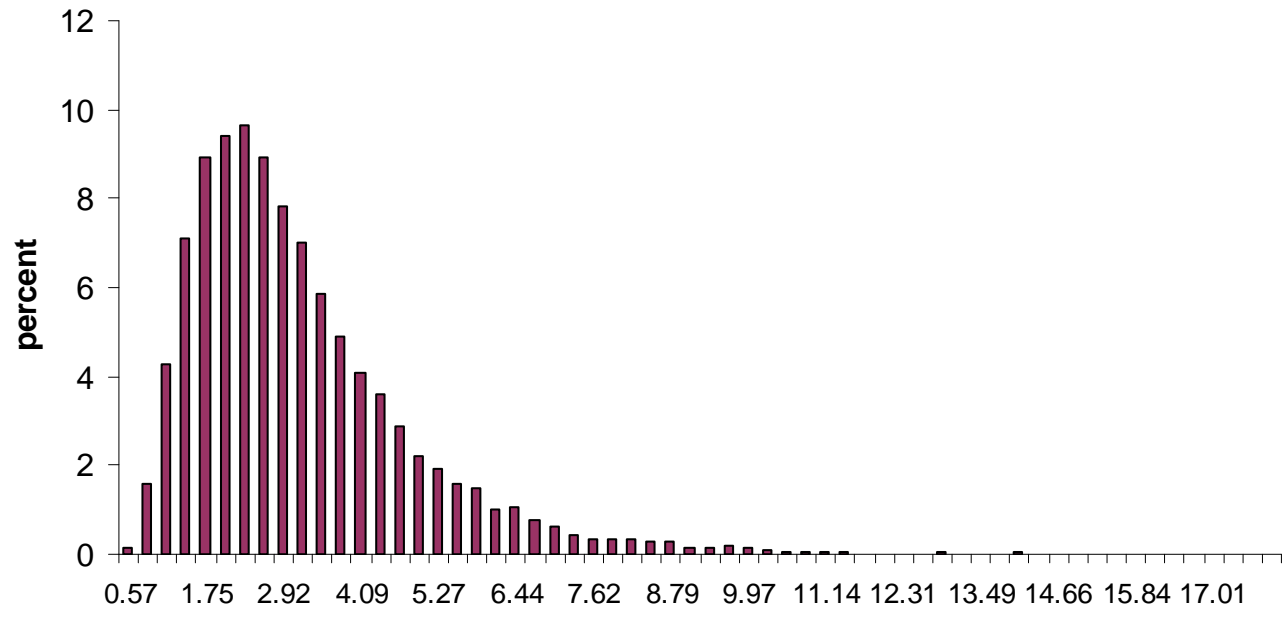


Figure 9

Losses under non-linear specification

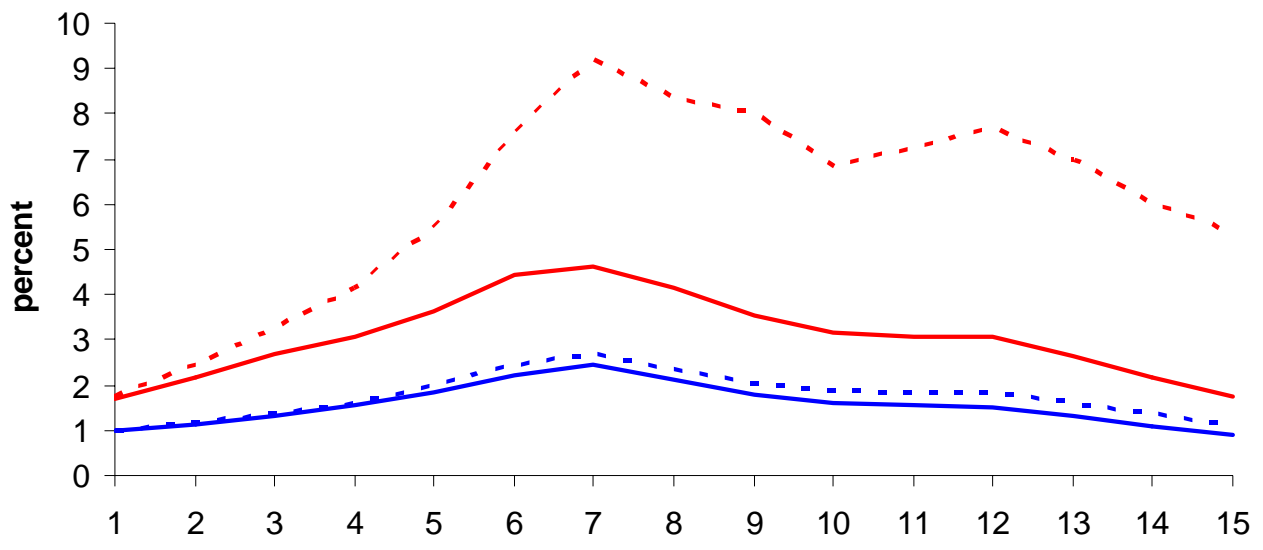

\begin{tabular}{ll}
\hline EL (no uncertainty) & \\
- - - EL (uncertainty) & - - - . 99.9\% VaR (no uncertainty)
\end{tabular}

\section{Figure 10}

Impact of the functional form on losses

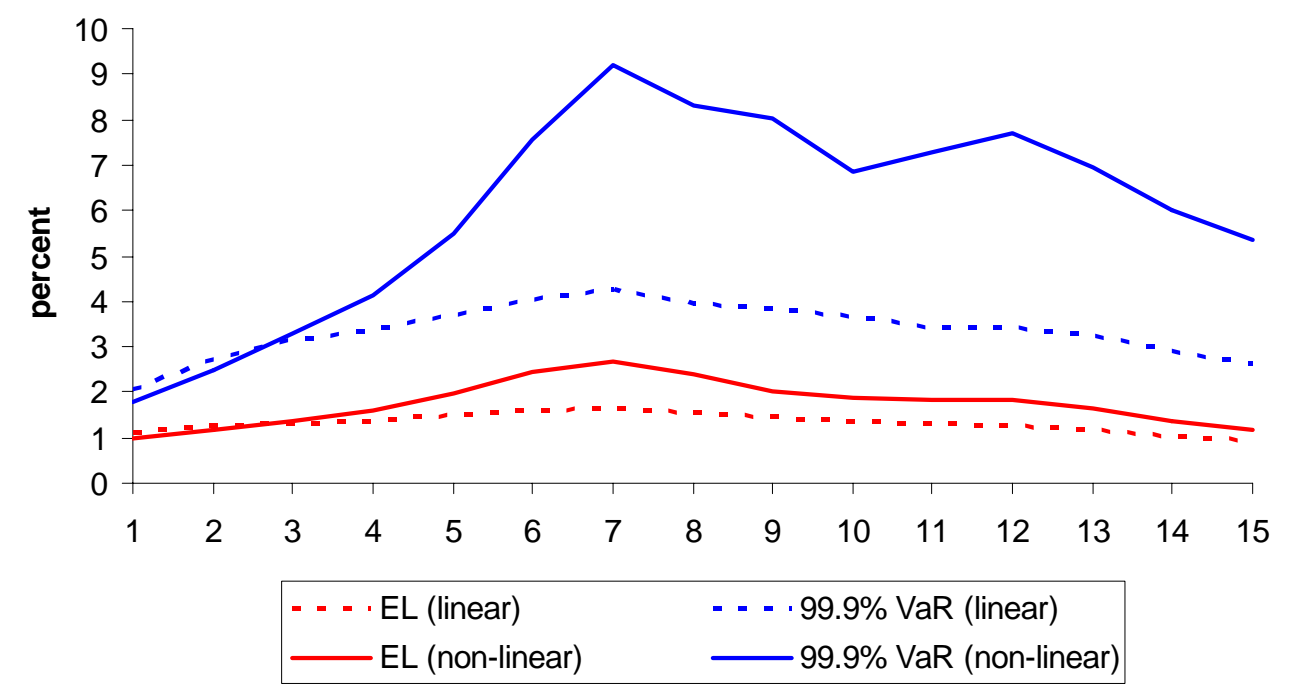




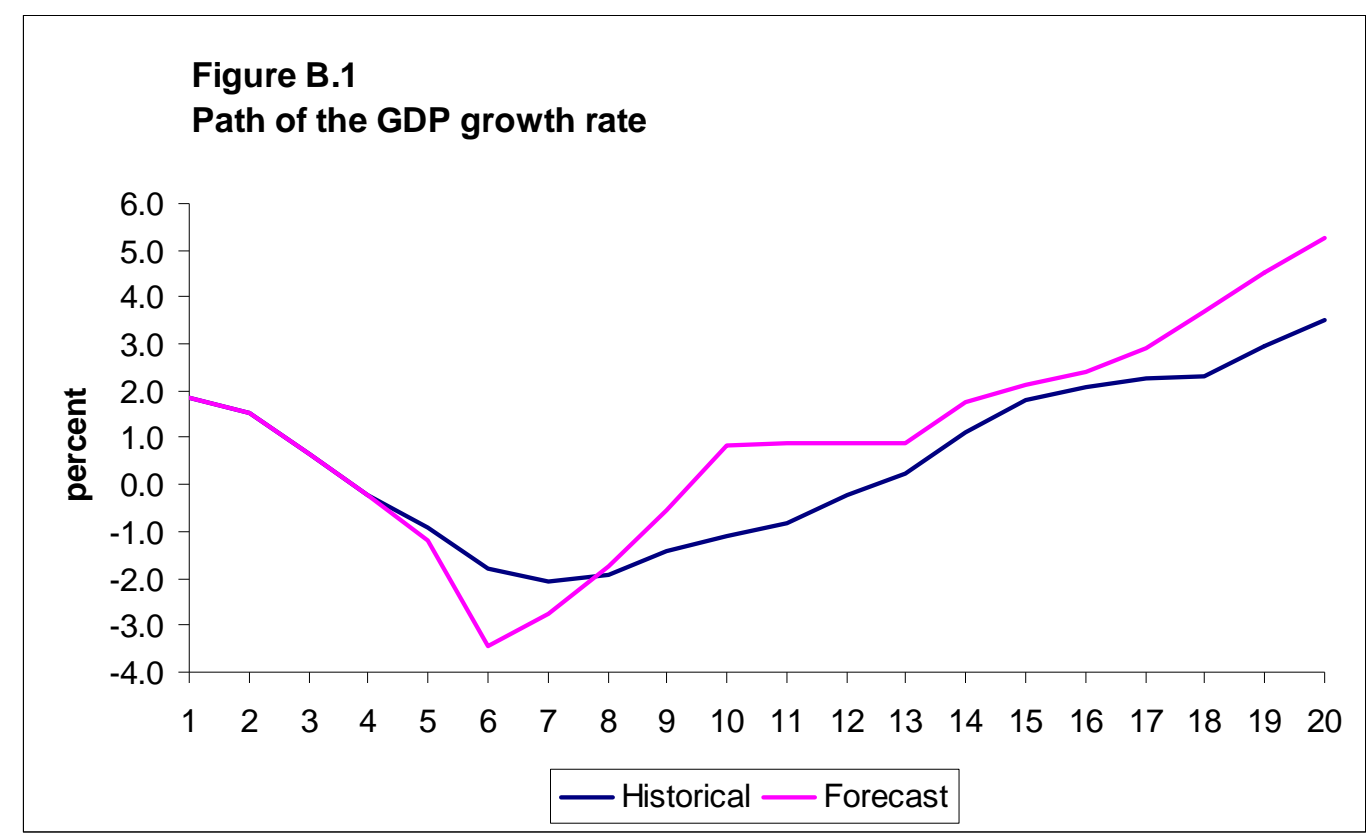

Figure B.2

Path of the interest rate

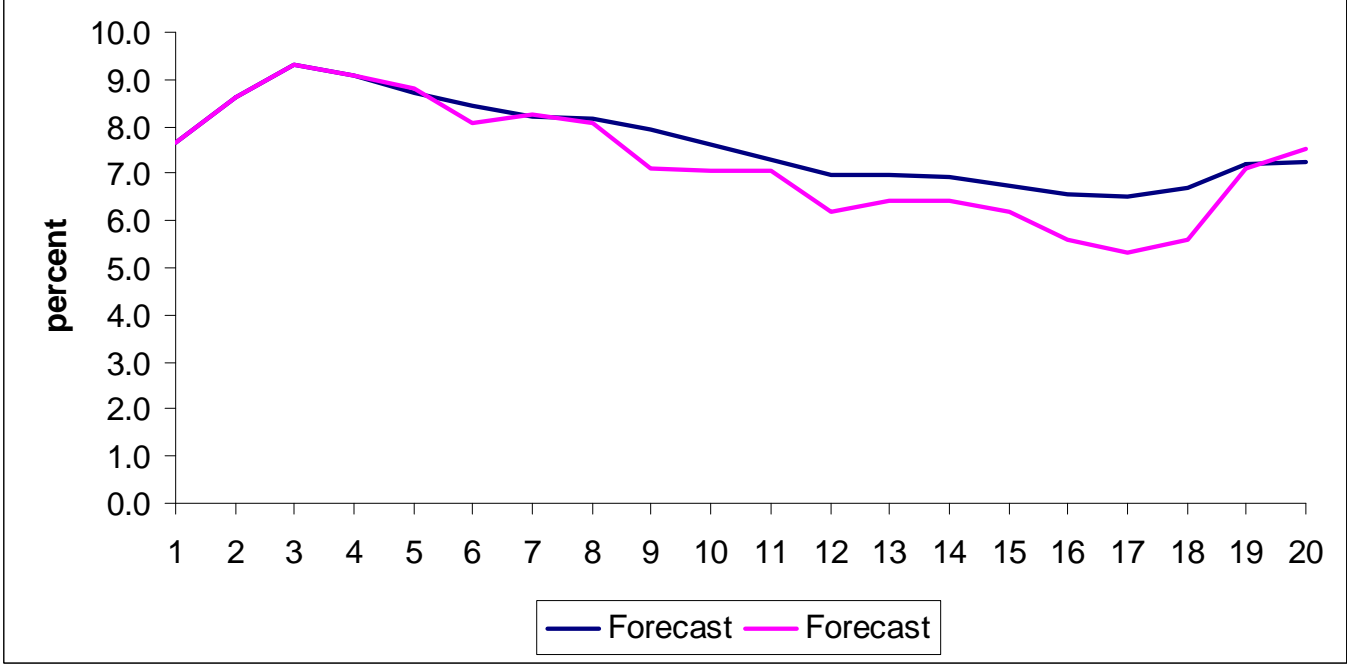




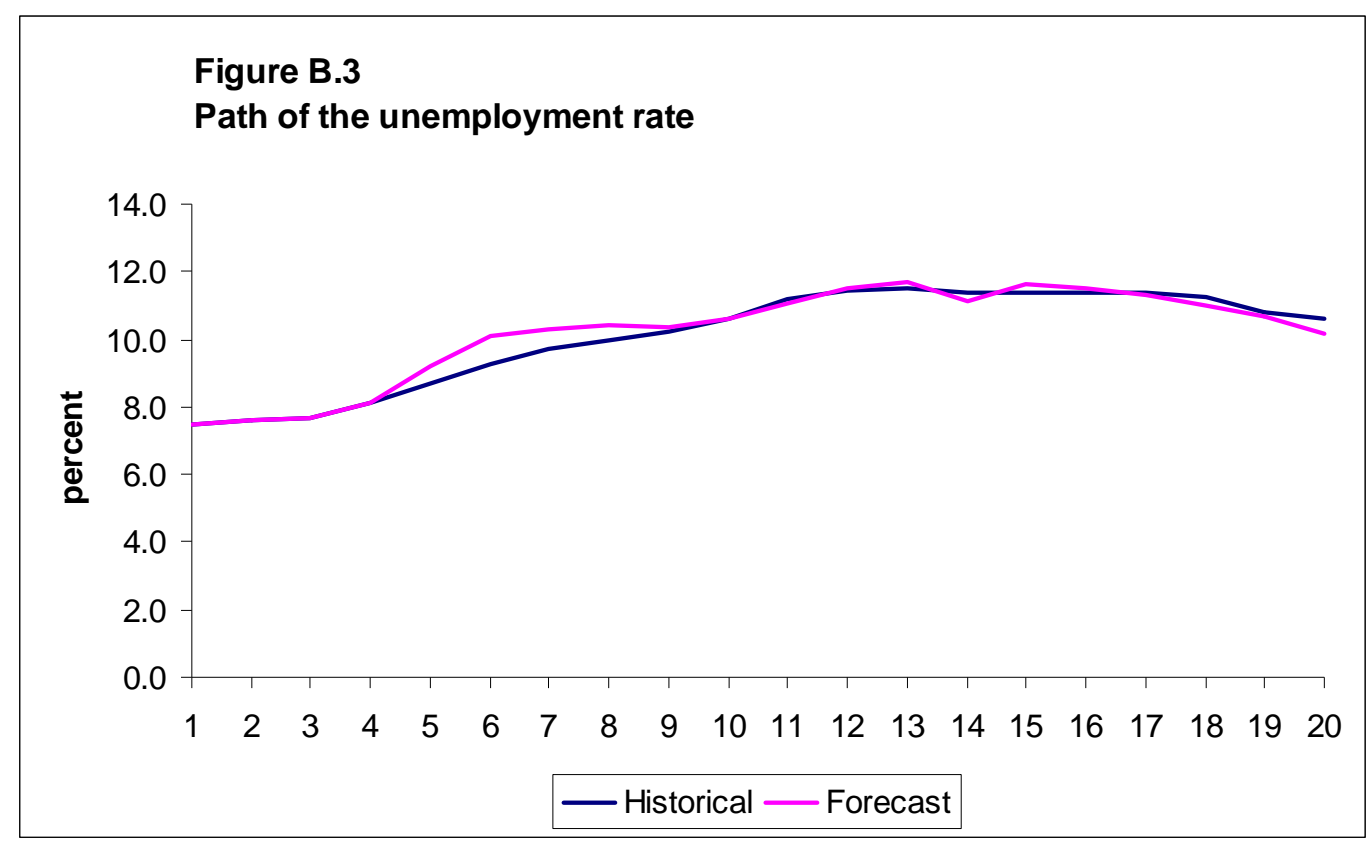

